Bull. Austral. Math. Soc.

$41 \mathrm{~A} 05,41 \mathrm{~A} 10,41 \mathrm{~A} 25$

VOL. 58 (1998) [505-512]

\title{
ON THE APPROXIMATION OF CERTAIN FUNCTIONS BY INTERPOLATING POLYNOMIALS
}

\author{
Michael Revers
}

In the present paper we consider the approximation of $|x|^{\alpha}$ on $[-1,1]$ by interpolating polynomials and we establish upper bounds for the approximation error. It turns out that these bounds, apart from the constants, are of the best possible order. We compare our results with estimates for the best approximation, established by Bernstein.

\section{INTRODUCTION}

Much attention has been devoted to the approximation of $|x|^{\alpha}$ by polynomials (Bell and Shah [1], Bernstein [2, 3], Byrne, Mills and Smith [6], Elosser [7, 8], Varga and Carpenter [11]) and by rational functions (Brutman and Passow [5], Newman [9], Stahl [10]). This work was motivated by Bernstein's result on the best approximation of $|x|^{\alpha}$ on $[-1,1]$. In 1938 Bernstein [3, p.186] established the following result: let $c_{j}$ be real for all $j, n \in N$ and denote $E_{n}(\alpha)=\left.\min _{c_{j}} \max _{-1 \leqslant x \leqslant 1}|| x\right|^{\alpha}-\left(c_{0}+c_{1} x+\cdots+c_{n} x^{n}\right) \mid$. Then we have the following

TheOREM 1. $\forall \alpha>0, \forall n \in N, n$ even, $n \geqslant n_{0}(\alpha)$ we have

$$
\begin{aligned}
\frac{C_{\alpha}}{n^{\alpha}}\left(\frac{\pi}{\pi+4}\right)^{\alpha} \frac{1}{2 \sqrt{2}} & \leqslant E_{n}(\alpha) \leqslant \frac{C_{\alpha}}{n^{\alpha}} \\
\text { with } C_{\alpha} & =\frac{4}{\pi}\left|\sin \pi \frac{\alpha}{2}\right| \int_{0}^{\infty} \frac{u^{\alpha-1}}{e^{u}+e^{-u}} d u
\end{aligned}
$$

Unfortunately, looking at Bernsteins proof, it seems to be difficult to find information concerning the size of $n_{0}(\alpha)$ (the case of an even integer $\alpha$ is an exceptional one, since in this case one can take $n \geqslant n_{0}(\alpha)=\alpha$ ) and moreover, the proof does not suggest how to give a simple construction of those approximating polynomials which will achieve the two-sided inequality. Let us bring to the reader's attention that, in the french translation of Bernsteins paper [3], and also in the russian original [4], the statement in Theorem 1 is not correctly presented. In these papers the condition $n \geqslant n_{0}(\alpha)$ is suppressed. In this paper we consider the explicit construction of certain interpolating polynomials on $|x|^{\alpha}$,

Received 2nd June, 1998

Copyright Clearance Centre, Inc. Serial-fee code: 0004-9729/98 \$A2.00+0.00. 
whose upper error bound for the approximation error does not exceed twice the value of the upper estimate in Theorem 1. Furthermore, the interpolating procedure works for all even $n \in N$.

\section{RESULTS}

Consider $F_{2 m}(\alpha)=\left.\max _{-1 \leqslant x \leqslant 1}|| x\right|^{\alpha}-R_{2 m}(x) \mid$, where $R_{2 m}(x)$ is the unique even interpolating polynomial of degree at most $2 m$ to $|x|^{\alpha}$ on $[-1,1]$ at the (Chebyshev) nodes $x_{0}=0, x_{j}=\cos (j-1 / 2) \pi / 2 m, j=1,2, \ldots, 2 m$. We shall prove the following:

ThEOREM 2. Let $n=2 m, m \in N, \alpha \in(0,2 / 3] \cup\{1\}$. Then we have

$$
F_{n}(\alpha)<\frac{2\left(\frac{2}{3}\right)^{1-\alpha}}{n^{\alpha}} .
$$

REMARK 3. (a) The proof of Theorem 2 is based on the convex behaviour of a certain function which is studied in the Appendix. For the possible extension of Theorem 2 to all $\alpha \in(0,1]$ the reader is referred to Remark 5 at the end of the paper.

(b) Before going into details of the proof, we present some numerical computations of the $C_{\alpha}$ 's from Theorem 1. (The numbers are rounded off to 2 digits.)

\begin{tabular}{|r|rrrrrrrrrr|}
\hline$\alpha$ & 0.1 & 0.2 & 0.3 & 0.4 & 0.5 & 0.6 & 0.7 & 0.8 & 0.9 & 1 \\
\hline$C_{\alpha}$ & 1.02 & 1.04 & 1.05 & 1.06 & 1.07 & 1.07 & 1.06 & 1.05 & 1.03 & 1 \\
$2(2 / 3)^{1-\alpha}$ & 1.39 & 1.45 & 1.51 & 1.57 & 1.63 & 1.70 & 1.77 & 1.84 & 1.92 & 2 \\
\hline
\end{tabular}

Proof: By the well known Lagrange interpolating formula one has

$$
R_{2 m}(x)=\sum_{j=0}^{2 m} f\left(x_{j}\right) L_{j}(x), L_{j}(x)=\prod_{\substack{i=0 \\ i \neq j}}^{2 m} \frac{x-x_{i}}{x_{j}-x_{i}}=\frac{w(x)}{\left(x-x_{j}\right) w^{\prime}\left(x_{j}\right)},
$$

with $w(x)=\prod_{j=0}^{2 m}\left(x-x_{j}\right)=x T_{2 m}(x)$, where $T_{m}(x)=\cos (m \arccos x)$ is the Chebyshev polynomial of first kind of degree $m$. For the indices $j=1,2, \ldots, 2 m$ we have

$$
w^{\prime}\left(x_{j}\right)=\cos \left[\left(j-\frac{1}{2}\right) \frac{\pi}{2 m}\right](-1)^{j+1} \frac{2 m}{\sin \left(j-\frac{1}{2}\right) \frac{\pi}{2 m}} .
$$


So we get

$$
\begin{aligned}
& R_{2 m}(x)=\sum_{j=1}^{2 m}\left|x_{j}\right|^{\alpha} \frac{x T_{2 m}(x)(-1)^{j+1} \sin \left(j-\frac{1}{2}\right) \frac{\pi}{2 m}}{\left(x-x_{j}\right) 2 m \cos \left(j-\frac{1}{2}\right) \frac{\pi}{2 m}} \\
&=\frac{x^{\alpha} T_{2 m}(x)}{2 m}\left[\sum_{j=1}^{m} \frac{(-1)^{j+1} \sin \left(j-\frac{1}{2}\right) \frac{\pi}{2 m}}{x-x_{j}} x_{j}^{\alpha-1} x^{1-\alpha}\right. \\
&\left.-\sum_{j=m+1}^{2 m} \frac{(-1)^{j+1} \sin \left(j-\frac{1}{2}\right) \frac{\pi}{2 m}}{x-x_{j}}\left(-x_{j}\right)^{\alpha-1} x^{1-\alpha}\right] .
\end{aligned}
$$

Now we look at

$$
x^{\alpha}=x^{\alpha} \frac{T_{2 m}(x)}{2 m} \sum_{j=1}^{2 m} \frac{(-1)^{j+1} \sin \left(j-\frac{1}{2}\right) \frac{\pi}{2 m}}{x-x_{j}},
$$

where the constant function 1 is exactly interpolated at the nodes $x_{1}, x_{2}, \ldots, x_{2 m}$. Combining formulas (1) and (2) we get for $0 \leqslant x \leqslant 1$ :

$$
\begin{aligned}
x^{\alpha}-R_{2 m}(x)=x^{\alpha} \frac{T_{2 m}(x)}{2 m} & {\left[\sum_{j=1}^{m} \frac{(-1)^{j+1} \sin \left(j-\frac{1}{2}\right) \frac{\pi}{2 m}}{x-x_{j}}\left(1-x_{j}^{\alpha-1} x^{1-\alpha}\right)\right.} \\
& \left.+\sum_{j=m+1}^{2 m} \frac{(-1)^{j+1} \sin \left(j-\frac{1}{2}\right) \frac{\pi}{2 m}}{x-x_{j}}\left(1+\left[-x_{j}\right]^{\alpha-1} x^{1-\alpha}\right)\right] .
\end{aligned}
$$

For the second sum in (3) we get

$$
\begin{gathered}
\sum_{j=m+1}^{2 m} \frac{(-1)(-1)^{3 m+1-j} \sin \left(3 m+\frac{1}{2}-j\right) \frac{\pi}{2 m}}{x-\cos \left(3 m+\frac{1}{2}-j\right) \frac{\pi}{2 m}} . \\
\quad\left(1+x^{1-\alpha}\left[-\cos \left(3 m+\frac{1}{2}-j\right) \frac{\pi}{2 m}\right]^{\alpha-1}\right) \\
=\sum_{j=1}^{m} \frac{(-1)^{j} \sin \left(j-\frac{1}{2}\right) \frac{\pi}{2 m}}{x+\cos \left(j-\frac{1}{2}\right) \frac{\pi}{2 m}}\left[1+x^{1-\alpha} \cos ^{\alpha-1}\left(j-\frac{1}{2}\right) \frac{\pi}{2 m}\right] .
\end{gathered}
$$


Let $t_{j}=(j-(1 / 2)) \pi / 2 m$ for $j=1,2, \ldots, m$. Combining (3) and (4) we obtain the approximation error for $x \geqslant 0$ :

$$
x^{\alpha}-R_{2 m}(x)=x^{\alpha} \frac{T_{2 m}(x)}{2 m} \sum_{j=1}^{m}(-1)^{j} \frac{\sin t_{j}}{\cos ^{1-\alpha} t_{j}}\left(\frac{x^{1-\alpha}+\cos ^{1-\alpha} t_{j}}{x+\cos t_{j}}+\frac{x^{1-\alpha}-\cos ^{1-\alpha} t_{j}}{x-\cos t_{j}}\right) .
$$

For $0<\alpha \leqslant 1,0 \leqslant x \leqslant 1,0 \leqslant t<\pi / 2$ we define

$$
h_{x}^{\alpha}(t)=\frac{\sin t}{\cos ^{1-\alpha} t}\left(\frac{x^{1-\alpha}+\cos ^{1-\alpha} t}{x+\cos t}+\frac{x^{1-\alpha}-\cos ^{1-\alpha} t}{x-\cos t}\right) .
$$

From the definition of $F_{2 m}(\alpha)$ we summarise

$$
\begin{gathered}
\qquad F_{2 m}(\alpha)=\left.\max _{-1 \leqslant x \leqslant 1}|| x\right|^{\alpha}-R_{2 m}(x)\left|=\max _{0 \leqslant x \leqslant 1} \frac{x^{\alpha}\left|T_{2 m}(x)\right|}{2 m}\right| H_{m}(x) \mid, \\
\text { with } H_{m}(x)=\sum_{j=1}^{m}(-1)^{j} h_{x}^{\alpha}\left(t_{j}\right), t_{j}=\left(j-\frac{1}{2}\right) \frac{\pi}{2 m} \text { and } j=1,2, \ldots, m .
\end{gathered}
$$

The essential analysis now depends on the estimate of $H_{m}(x)$. We consider the case $m=2 k, k \in N$ and we write

$$
H_{m}(x)=\int_{t_{1}}^{t_{2}} h_{x}^{\prime \alpha}(t) d t+\int_{t_{3}}^{t_{4}} h_{x}^{\prime \alpha}(t) d t+\cdots+\int_{t_{m-1}}^{t_{m}} h_{x}^{\prime \alpha}(t) d t .
$$

Let us assume $h_{x}^{\prime \alpha}(t)$ to be non negative and increasing in $t \in[0, \pi / 2), \alpha \in(0,2 / 3] \cup\{1\}$, $0 \leqslant x \leqslant 1$ (the proof of this appears in the appendix). We have the following lower and upper bounds for $H_{m}(x)$ :

$$
\begin{aligned}
& 2 H_{m}(x) \leqslant \int_{\pi / 4 m}^{\pi / 2-\pi / 4 m} h_{x}^{\alpha \alpha}(t) d t+\int_{\pi / 2-3 \pi / 4 m}^{\pi / 2-\pi / 4 m} h_{x}^{\prime \alpha}(t) d t, \\
& 2 H_{m}(x) \geqslant \int_{\pi / 4 m}^{3 \pi / 4 m} h_{x}^{\prime \alpha}(t) d t+\int_{\pi / 4 m}^{\pi / 2-\pi / 4 m} h_{x}^{\prime \alpha}(t) d t .
\end{aligned}
$$

The case $m=2 k+1, k \in N$ is treated similarly, and we establish the following bounds: CASE: $m=2 k$.

$$
\begin{gathered}
h_{x}^{\alpha}\left(\frac{\pi}{2}-\frac{\pi}{4 m}\right)+h_{x}^{\alpha}\left(\frac{3 \pi}{4 m}\right)-2 h_{x}^{\alpha}\left(\frac{\pi}{4 m}\right) \leqslant 2 H_{m}(x), \\
2 H_{m}(x) \leqslant 2 h_{x}^{\alpha}\left(\frac{\pi}{2}-\frac{\pi}{4 m}\right)-h_{x}^{\alpha}\left(\frac{\pi}{2}-\frac{3 \pi}{4 m}\right)-h_{x}^{\alpha}\left(\frac{\pi}{4 m}\right) .
\end{gathered}
$$

CASE: $m=2 k+1$. 


$$
\begin{gathered}
-2 h_{x}^{\alpha}\left(\frac{\pi}{2}-\frac{\pi}{4 m}\right)+h_{x}^{\alpha}\left(\frac{\pi}{2}-\frac{3 \pi}{4 m}\right)+h_{x}^{\alpha}\left(\frac{3 \pi}{4 m}\right)-2 h_{x}^{\alpha}\left(\frac{\pi}{4 m}\right) \leqslant 2 H_{m}(x), \\
2 H_{m}(x) \leqslant-h_{x}^{\alpha}\left(\frac{\pi}{2}-\frac{\pi}{4 m}\right)-h_{x}^{\alpha}\left(\frac{\pi}{4 m}\right) .
\end{gathered}
$$

By the assumptions on $h_{x}^{\alpha \alpha}(t)$ (note that $h_{x}^{\alpha}(t)$ is increasing) and since $h_{x}^{\alpha}(t) \geqslant 0$ for all $t$ (to be seen from (5)), we get the somewhat weaker estimate

$$
\left|H_{m}(x)\right| \leqslant h_{x}^{\alpha}\left(\frac{\pi}{2}-\frac{\pi}{4 m}\right) \forall m \in N, 0 \leqslant x \leqslant 1, \alpha \in\left(0, \frac{2}{3}\right] \cup\{1\} .
$$

Using (7) we are able to establish Theorem 2. Again we use (5) to show that

$$
h_{x}^{\alpha}(t)=\frac{2}{x^{\alpha}} \frac{\sin t}{\cos ^{1-\alpha} t}\left[1-\frac{\cos ^{2} t}{\cos ^{\alpha} t} \frac{x^{\alpha}-\cos ^{\alpha} t}{x^{2}-\cos ^{2} t}\right]
$$

and we estimate

$$
0 \leqslant h_{x}^{\alpha}(t) \leqslant \frac{2}{x^{\alpha}} \frac{\sin t}{\cos ^{1-\alpha} t} \text { for } 0<x \leqslant 1, \alpha \in\left(0, \frac{2}{3}\right] \cup\{1\}, 0 \leqslant t<\frac{\pi}{2} .
$$

We have to evaluate $h_{x}^{\alpha}(t)$ at $t_{m}=\pi / 2-\pi / 4 m$. So let $\rho_{m}=(\pi / 2)-t_{m}$ and note that $0<$ $\rho_{m}<\pi / 2<\sqrt{6}$ for all $m \geqslant 1$. Then $\cos t_{m}=\cos \left((\pi / 2)-\rho_{m}\right)=\sin \rho_{m}>\rho_{m}-\rho_{m}^{3} / 6>0$. Since $\rho_{m}-\rho_{m}^{3} / 6=(\pi / 4 m)\left(1-\left(\pi^{2} / 96 m^{2}\right)\right)>3 / 4 m$ for all $m \geqslant 2$, we estimate (8) to $0 \leqslant h_{x}^{\alpha}\left(t_{m}\right) \leqslant\left(2 / x^{\alpha}\right)\left(\sin \left(t_{m}\right)\right) /\left(\cos ^{1-\alpha}\left(t_{m}\right)\right)<\left(2 / x^{\alpha}\right)(4 m / 3)^{1-\alpha}$. Inserting in (7) gives

$$
\left|H_{m}(x)\right|<\frac{2}{x^{\alpha}} \frac{(2 m)^{1-\alpha} 2^{1-\alpha}}{3^{1-\alpha}} .
$$

Since $\left|T_{n}(x)\right| \leqslant 1$ for $-1 \leqslant x \leqslant 1, n \in N$, we combine (6) and (9) to prove Theorem 2 for all $n=2 m, m \geqslant 2$. For the remaining case $n=2$ one simply shows that $F_{2}(\alpha)=$ $\left.|| x\right|^{\alpha}-R_{2}(x) \mid \leqslant \sqrt{2}^{-\alpha}$. So our estimate holds for all $n=2 m, m \in N$, and the proof is finished.

\section{APPENDIX}

Again let $\alpha \in(0,2 / 3] \cup\{1\}, 0 \leqslant x \leqslant 1$ and $0 \leqslant t<\pi / 2$. We have to show that $h_{x}^{\alpha}(t) \geqslant 0, h_{x}^{\prime \alpha}(t) \geqslant 0$ and $h_{x}^{\prime \alpha}(t) \geqslant 0$. We write $(5)$ in the form $h_{x}^{\alpha}(t):=g_{1}(t)+g_{2}(t)$ (for convenience we omit the indices $x$ and $\alpha$ ):

$$
\begin{aligned}
& g_{1}(t)=\frac{\sin t}{x+\cos t}\left[1+\left(\frac{x}{\cos t}\right)^{1-\alpha}\right], \\
& g_{2}(t)=\frac{\sin t}{\cos ^{1-\alpha} t} \frac{x^{1-\alpha}-\cos ^{1-\alpha} t}{x-\cos t} .
\end{aligned}
$$


We begin with $g_{1}$. One simply shows that $(i) g_{1}$ is non negative, $(i i) g_{1}^{\prime}$ is non negative and (iii) $g_{1}^{\prime}$ is increasing. Note that the restriction of $\alpha \in(0,2 / 3] \cup\{1\}$ can be extended to $\alpha \in(0,1]$ without loss of $(i),(i i)$ and $(i i i)$. The more problem arises with $g_{2}$. For $\alpha=1$ there is nothing to show. For the remaining case $\alpha \in(0,2 / 3]$ we study

$$
g(y):=\frac{y^{1-\alpha}-x^{1-\alpha}}{y-x}, 0<y \leqslant 1,0 \leqslant x \leqslant 1 .
$$

We have the following

LEMMA 4.

(i) $g(y) \geqslant 0,0<y \leqslant 1,0<\alpha \leqslant 1$,

(ii) $g^{\prime}(y) \leqslant 0,0<y \leqslant 1,0<\alpha \leqslant 1$,

(iii) $g^{\prime \prime}(y) \geqslant 0,0<y \leqslant 1,0<\alpha \leqslant 1$.

PROOF:

(i) This is trivial.

(ii) By identifying $g^{\prime}(x)$ with $\lim _{y \rightarrow x} g^{\prime}(y)$ one can easily establish

$$
g^{\prime}(y)= \begin{cases}\frac{\frac{1-\alpha}{y^{\alpha}}-\frac{y^{1-\alpha}-x^{1-\alpha}}{y-x}}{y-x} & \text { for } y \neq x, \\ \lim _{y \rightarrow x} g^{\prime}(y)=-\frac{\alpha(1-\alpha)}{2 x^{1+\alpha}} & \text { for } y=x .\end{cases}
$$

CASE A: $y=x$. Then $g^{\prime}(x) \leqslant 0$.

CASE B: $y>x$. Then one has

$$
\frac{y^{1-\alpha}-x^{1-\alpha}}{y-x} \geqslant \frac{1-\alpha}{y^{\alpha}}
$$

and inserting in $(10)$, we get $g^{\prime}(y) \leqslant 0$.

Case C: $y<x$. Analoguous to Case B one has

$$
\frac{y^{1-\alpha}-x^{1-\alpha}}{y-x} \leqslant \frac{1-\alpha}{y^{\alpha}}
$$

and again, by inserting in $(10)$, we get $g^{\prime}(y) \leqslant 0$.

(iii) For convenience we again identify $g^{\prime \prime}(x)$ with its limit $\lim _{y \rightarrow x} g^{\prime \prime}(y)$. Then one simply gets

$$
g^{\prime \prime}(y)= \begin{cases}2 \frac{y^{1-\alpha}-x^{1-\alpha}}{(y-x)^{3}}-\frac{2(1-\alpha)}{y^{\alpha}(y-x)^{2}}-\frac{\alpha(1-\alpha)}{y^{1+\alpha}(y-x)} & \text { for } y \neq x \\ \lim _{y \rightarrow x} g^{\prime \prime}(y)=\frac{\alpha(1+\alpha)(1-\alpha)}{3 x^{2+\alpha}} & \text { for } y=x\end{cases}
$$


and a similar treatment to that in (ii) gives the required properties. The proof is finished.

From Lemma 4 we deduce that the function

$$
f_{2}(t):=g(\cos (t))=\frac{x^{1-\alpha}-\cos ^{1-\alpha} t}{x-\cos t}
$$

is non negative, increasing and convex for $0 \leqslant t<\pi / 2,0<\alpha \leqslant 1$ and $0 \leqslant x \leqslant 1$. Now we shall study the function $g_{2}$. We write

$$
g_{2}(t)=f_{1}(t) \cdot f_{2}(t)=\frac{\sin t}{\cos ^{1-\alpha} t} \frac{x^{1-\alpha}-\cos ^{1-\alpha} t}{x-\cos t} .
$$

Then $g_{2}$ is non negative and

$$
g_{2}^{\prime}(t)=f_{1}^{\prime}(t) f_{2}(t)+f_{1}(t) f_{2}^{\prime}(t) \geqslant 0 \text { if } f_{1}^{\prime}(t) \geqslant 0 .
$$

But this is the case for $0 \leqslant t<\pi / 2,0<\alpha \leqslant 1$. Next we study $g_{2}^{\prime \prime}(t)$. One sees simply

$$
g_{2}^{\prime \prime}(t) \geqslant 0 \text { if } f_{1}^{\prime \prime}(t) \geqslant 0
$$

An easy calculation shows that

$$
f_{1}^{\prime \prime}(t)=\frac{d^{2}}{d t^{2}}\left[\frac{\sin t}{\cos ^{1-\alpha} t}\right] \geqslant 0 \text { for all } 0 \leqslant t<\frac{\pi}{2} \text { if and only if } \alpha \leqslant \frac{2}{3} .
$$

Hence we conclude $h_{x}^{\alpha}(t)$ is non negative, increasing and convex for all $0 \leqslant t<\pi / 2,0 \leqslant$ $x \leqslant 1$ and $\alpha \in(0,2 / 3] \cup\{1\}$.

REMARK 5. There is a strong evidence that the function $g_{2}$ is also convex for $2 / 3<$ $\alpha \leqslant 1$ and thus Theorem 2 holds for all $0<\alpha \leqslant 1$. I have not found a proof of this as yet, and so we finish with the following:

Conjecture 6 . Let $0 \leqslant x \leqslant 1,0<\alpha \leqslant 1$. Then

$$
f_{x}^{\alpha}(t)=\frac{\sin t}{\cos ^{1-\alpha} t} \frac{x^{1-\alpha}-\cos ^{1-\alpha} t}{x-\cos t}
$$

is convex on $[0, \pi / 2)$.

\section{REFERENCES}

[1] R.A. Bell and S.M. Shah, 'Oscillating polynomials and approximation to fractional powers of $x^{\prime}, J$. Approx. Theory 1 (1968), 269-274.

[2] S. Bernstein, 'Sur la meilleure approximation de $|x|$ par des polynômes de degrés donnés', Acta Math. 37 (1913), 1-57.

[3] S. Bernstein, 'Sur la meilleure approximation de $|x|^{p}$ par des polynômes de degrés trés élevés', Bull. Acad. Sci. USSR Sér. Math. 2 (1938), 181-190. 
14] S. Bernstein, 'On the best approximation of $|x|^{p}$ by means of polynomials of extremely high degree', (in Russian), Izv. Akad. Nauk. SSSR 2 (1938), 169-180.

[5] L. Brutman and E. Passow, 'Rational interpolation to $|x|$ at the Chebyshev nodes', Bull. Austral. Math. Soc. 56 (1997), 81-86.

[6] G.J. Byrne, T.M. Mills and S.J. Smith, 'On Lagrange interpolation with equidistant nodes', Bull. Austral. Math. Soc. 42 (1990), 81-89.

[7] P.D. Elosser, 'Approximation of powers of $x$ by polynomials', $J$. Approx. Theory 23 (1978), 163-174.

[8] P.D. Elosser, 'Chebychev coefficients in approximation of powers of $x$ ', J. Approx. Theory 63 (1990), 293-302.

[9] D.J. Newman, 'Rational approximation to $|x|$ ', Michigan Math. J. 11 (1964), 11-14.

[10] H. Stahl, 'Best uniform rational approximation of $x^{\alpha}$ on $[0,1]$ ', Bull. Amer. Math. Soc. 28 (1993), 116-122.

[11] R.S. Varga and A.J. Carpenter, 'On the Bernstein Conjecture in approximation theory', Const. Approx. 1 (1985), 333-348.

Department of Mathematics

University of Salzburg

Hellbrunnerstrasse 34

A-5020 Salzburg

Austria

e-mail: Michael.Revers@sbg.ac.at 